\title{
The Integrated Web Portal for Escalation with Overdose Control (EWOC)
}

\author{
Haibin Wang ${ }^{*}$, Mourad Tighiouart, Shao-Chi Huang, Dror Berel, Galen Cook-Wiens, \\ Catherine Bresee, Quanlin Li and André Rogatko
}

\begin{abstract}
Biostatistics \& Bioinformatics Research Center, Cedars-Sinai Medical Center, 116 N. Robertson Blvd, Los Angeles, CA 90048, USA
\end{abstract}

\begin{abstract}
In this paper, we present the design and implementation of a novel web portal for the cancer phase I clinical trial design method Escalation with Overdose Control (EWOC). The web portal has two major components: a web-based dose finding calculator; and a standalone and downloadable dose finding software which can be installed on Windows operating systems. The web-based dose finding calculator uses industry standards and is a database-driven and distributed computing platform for designing and conducting dose finding in cancer phase I clinical trials utilizing EWOC methodology. The web portal is developed using open source software: PHP, JQuery, R and OpenBUGS. It supports any standard browsers with internet connection. The web portal can be accessed at: http://biostatistics.csmc.edu.
\end{abstract}

Keywords: EWOC, Bayesian method, cancer phase I clinical trial, maximum tolerated dose, open source.

\section{INTRODUCTION}

Phase I clinical trials are the initial introduction of a new investigational drug into human subjects [1]. In this phase, the safety, tolerability, pharmacokinetics and pharmacodynamics of a drug will be first assessed on a small group of volunteers. In contrast to healthy volunteers used in other phase I trials, participants in cancer phase I clinical trials are patients at advanced cancer stages who consent to enroll in the trial as a last resort in seeking cure given the inherent toxicity of oncology drugs [2]. Thus, cancer phase I trials should be carefully designed to not only achieve therapeutic benefits by avoiding low, non-therapeutic doses, but also monitor the severity of side-effects via controlling toxic overdoses [3].

Escalation with Overdose Controls (EWOC) was the first dose-finding procedure to directly incorporate the ethical constraint of minimizing the chance of treating patients at unacceptably high doses. Its defining property is that the expected proportion of patients treated at doses above the maximum tolerated dose (MTD) is equal to a specified value $\alpha$, the feasibility bound. This value is selected by the clinician and reflects the estimated level of concern about overdosing. Zacks et al. [4] showed that among designs with this defining property, EWOC minimizes the average amount by which patients are underdosed. This means that EWOC approaches the MTD as rapidly as possible, while keeping the expected proportion of patients overdosed less than the value $\alpha$. Zacks et al. [4] also showed that, as a trial progresses, the dose sequence defined by EWOC approaches the MTD (i.e., the sequence of recommended doses converges in probability to the MTD). Eventually, all

*Address correspondence to this author at the Biostatistics \& Bioinformatics Research Center, Cedars-Sinai Medical Centre, 116 N. Robertson Blvd, Los Angeles, CA 90048, USA; Tel: 310-423-3315; Fax: 310-423-3315;

E-mail: Haibin.wang @cshs.org patients beyond a certain time would be treated at doses sufficiently close to the MTD.

Babb and Rogatko [5] provide a summary of Bayesian phase I design methods and Tighiouart et al. [6] studied the performance of EWOC under a rich class of prior distributions for design parameters. Tighiouart and Rogatko [7] have demonstrated that EWOC is coherent. Software implementations include EWOC 1.0beta, published in 1988 by Rogatko and Babb, and EWOC 2.0 in 2005 by $\mathrm{Xu}$, Tighiouart and Rogatko. In the past 14 years, EWOC has become a very successful tool to help clinicians design cancer phase I clinical trials. EWOC has been used to design phase I studies approved by the Research Review Committees and the Institute Review Boards of many institutions such as Fox Chase Cancer Center (Philadelphia, PA), Winship Cancer Institute (Atlanta, GA), and Samuel Oschin Comprehensive Cancer Institute (Los Angeles, CA).

Here we present the design and implementation of a novel web portal using EWOC methodology for cancer phase I clinical trials. The web portal consists of two major components: a web-based online dose finding calculator and a standalone software. The standalone software version 3.1.3 was developed by Cook-Wiens, Tighiouart and Rogatko. End users can access the web page at http://biostatistics. csmc.edu/ewoc/ewoc-s.php using any standard web browser. After submitting registration information, the download process will automatically save the software on the user's personal computer. The web-based online dose finding calculator is based on industry standards and is a databasedriven and distributed computing platform for designing and conducting dose finding in cancer phase I clinical trials utilizing EWOC methodology. The web portal is developed using open source software: PHP, JQuery, R and OpenBUGS. It supports any standard internet web browser and concurrent users. In order to use the web-based calculator, the user is required to create an account first at: 
http://biostatistics.csmc.edu/ewoc/register.php. After that, the user can login into the system to conduct dose finding.

In this paper we will briefly introduce the methodology used in EWOC. Then the architecture and system workflow will be elaborated to display its full functionality. Then, we provide an example to demonstrate the usage of the webbased online dose finding calculator. And in our concluding remarks we will also provide direction for future work.

\section{METHODS}

The main purpose of a cancer phase I clinical trial is to determine the dose level of a new treatment regimen to be adopted in the next phase II clinical trials. It is generally assumed for cancer therapy that toxicity is a prerequisite for optimal anti-tumour activity [8]. While the new treatment regimen may produce therapeutic benefits to patients, it may also have toxic reactions. Higher therapeutic outcomes can be achieved by increasing the dose which may result in a higher risk of severe toxicity to patients. Meanwhile, the use of low and non-therapeutic dose levels should be minimized to prevent the occurrence of drug-resistance in patients. Therefore, the goal is to determine the MTD, defined as the dose expected to cause certain level of medical unacceptable, dose limiting toxicity (DLT) in a specified proportion $\theta$ of patients [9]:

$$
\operatorname{Pr} o b\{D L T \mid \text { Dose }=M T D\}=\theta .
$$

In other words, a proportion $\theta$ of patients will show DLT if the population of patients is treated at MTD. The parameter $\theta$ is usually set between 0.1 and 0.5 . If the DLT is a transient, correctable or non-fatal condition, $\theta$ would be set relatively high, otherwise, set relatively low when it is lethal or life threatening [2].

The key concept underlying EWOC is that one can select dose level for use in a phase I trial so that the predicted proportion of patients receiving a dose level above the MTD is equal to a specified value $\alpha$, defined as the feasibility bound [2]:

$\operatorname{Pr} o b\left\{M T D \leq x_{k} \mid D_{k}\right\}=\alpha$

Here $x_{k}$ denotes the dose level for the $k^{\text {th }}$ patient and $D_{k}$ includes the information for dose, toxicity for the $k^{\text {th }}$ patient and all previously treated patients. This guarantees that the predicted probability that the given dose for each patient exceeds the MTD is equal to $\alpha$. The value of $\alpha$ can be gradually increased in the program. As the trial progress, the accrued knowledge of the target dose invokes the need of a relatively higher probability of exceeding target dose.

\subsection{Dose-Toxicity Model}

A two parameter logistic based model was considered as the dose-toxicity relationship in EWOC:

$$
\operatorname{Pr} o b\{D L T \mid \text { Dose }=x\}=\frac{\exp \left(\beta_{0}+\beta_{1} x\right)}{1+\exp \left(\beta_{0}+\beta_{1} x\right)}
$$

where $\beta_{0}$ and $\beta_{1}$ are the unknown location and scale parameters and $x$ represents the dose level. The above location-scale family model is reparameterized in terms of the MTD $\gamma$ and the probability of DLT at initial dose $\rho_{0}$, parameters clinicians can easily interpret [3]. It can be shown [2] that the likelihood based on model (3) expressed in terms of $\gamma$ and $\rho_{0}$ is

$\mathrm{L}\left(\rho_{0}, \gamma \mid \mathrm{D}_{\mathrm{k}}\right)=\frac{\prod_{i \in S(k)} \exp \left\{f\left(\rho_{0}, \gamma \mid \mathrm{D}_{\mathrm{i}}\right)\right\}}{\prod_{i=1}^{m(k)}\left[1+\exp \left\{f\left(\rho_{0}, \gamma \mid \mathrm{D}_{\mathrm{i}}\right)\right\}\right]}$

where

$f\left(\rho_{0}, \gamma \mid \mathrm{D}_{\mathrm{i}}\right)=\frac{\left(\gamma-x_{i}\right) \log \left(\frac{\rho_{0}}{1-\rho_{0}}\right)+\left(x_{i}-X_{\min }\right) \log \left(\frac{\theta}{1-\theta}\right)}{\gamma-X_{\min }}$

$S(k)=\left\{i=1, \ldots, m(k): y_{i}=1\right\}$, and $m(k)$ are the number of observations available at the time of treatment for the $k^{\text {th }}$ patient. $X_{\min }$ and $X_{\max }$ stand for the minimum and maximum dose levels in the trial.

\subsection{Dose Escalation Method}

By combining prior information of $\gamma, \rho_{0}$ and the likelihood of all the previous patients available, the joint posterior distribution of $\left(\gamma, \rho_{0}\right)$ will be obtained. Integrating out $\rho_{0}$, the marginal posterior distribution of the MTD will be available.

$\pi_{k}(x)=\int_{X \min }^{x} \int_{0}^{\theta} P\left(\rho_{0}, \gamma \mid D_{k}\right) d \rho_{0} d \gamma$.

where

$P\left(\rho_{0}, \gamma \mid D_{k}\right)=\tau^{-1} L\left(\rho_{0}, \gamma \mid D_{k}\right) I_{[0, \theta] \times\left[X_{\min , X_{\max }}\right.}\left(\rho_{0}, \gamma\right)$

The dose to be allocated to the $(k+1)^{\text {st }}$ patient is the dose level $x_{k+1}$ that ensure

$\pi_{k}\left(x_{k+1}\right)=\alpha$, where $\alpha$ is the feasibility bound.

\subsection{Estimation of the MTD}

At the conclusion of the trial, the estimate of the MTD is given by [3]:

$\hat{\gamma}_{l}=\underset{x \in\left[X_{\min ,}, X_{\max }\right]}{\arg \inf } \int_{X \min }^{X \max } l_{\alpha}(x, \gamma) \pi\left(\gamma \mid D_{n}\right) d \gamma$

where $\pi\left(x \mid D_{n}\right)$ is the posterior density of the MTD given all the observed data from the phase I trial and $l_{\alpha}$ is an asymmetric loss function given by

$l_{\alpha}(x, \gamma)=\left\{\begin{array}{l}\alpha(\gamma-x) \text { if } x \leq \gamma \text { that is, if } x \text { is an underdose } \\ (1-\alpha)(x-\gamma) \text { if } x>\gamma \text { that is, if } x \text { is an overdose. }\end{array}\right.$

\section{WEB PORTAL DEVELOPMENT}

The web-based online dose finding calculator is based on standard three tier architecture (Fig. 1). The front end is implemented in open source software PHP and JQuery. JQuery provides an excellent user input validation. The EWOC core algorithm is realized in open source software $\mathrm{R}$ and OpenBUGS. The middleware is also implemented in 


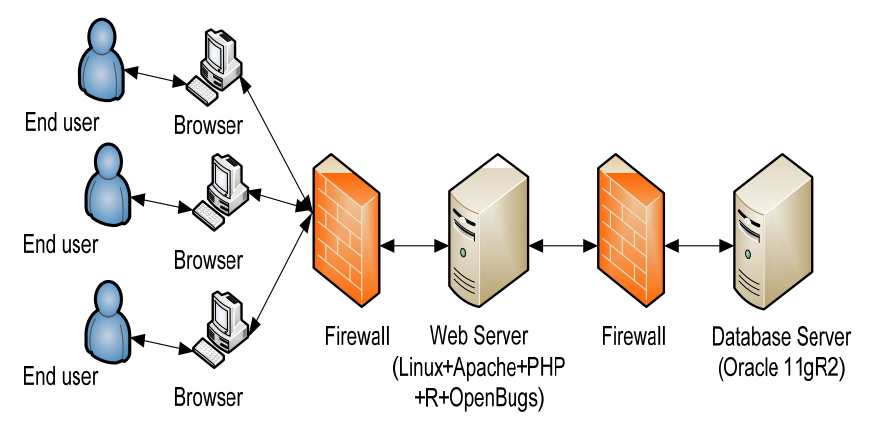

Fig. (1). High level architecture of web-based online calculator.

PHP to bridge front end interface with $\mathrm{R}$ implementation. The backend database is Oracle $11 \mathrm{gR} 2$.

The web-based online dose finding calculator is database-driven, client/server, multi-user based architecture. One new feature of this web-based online calculator is that eachend user creates a password-protected account prior to use. After logging in into the web portal, the user can design and conduct dose finding with all results stored in the database. All results are retrievable by the user who created each project and task. New projects can be started or previously stored ones can be resumed by the user at each

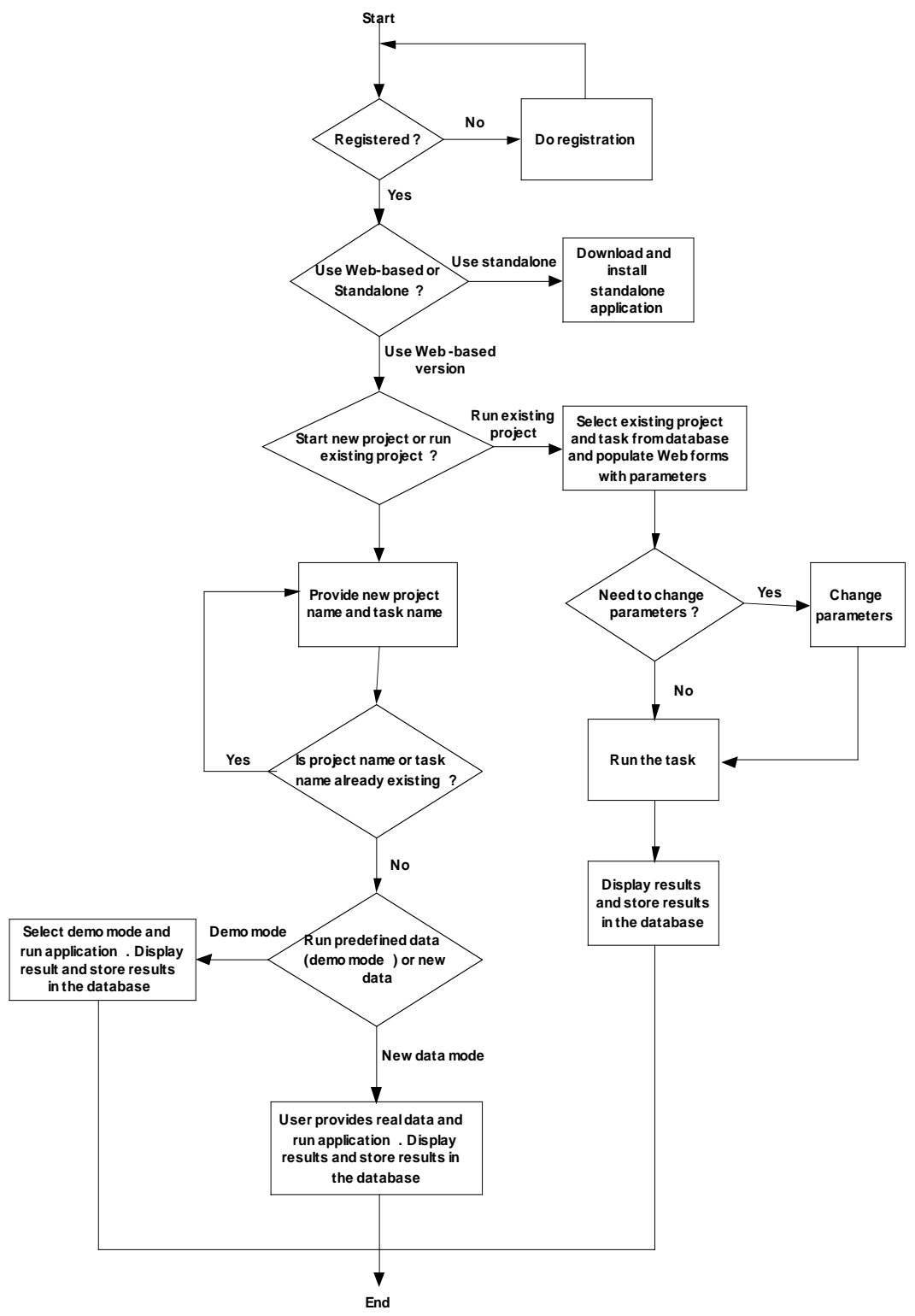

Fig. (2). System workflow of web-based online calculator. 
login. Fig. (2) shows the system workflow of the web-based online dose finding calculator.

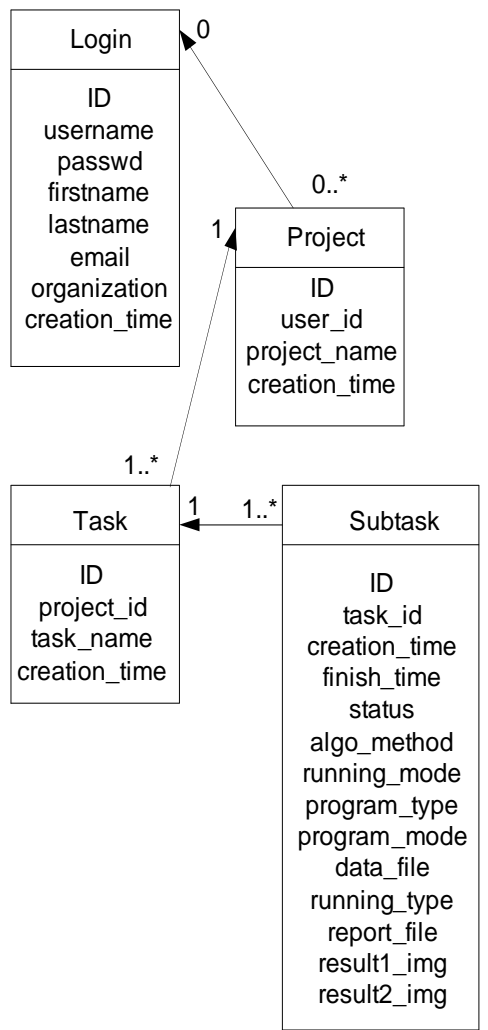

Fig. (3). ER diagram of the core tables of the web portal.

There are six database tables to store users' profile, projects, tasks, subtasks, and parameter information. The ER diagram of the core tables of the system is displayed in Fig. (3).

The web-based online calculator also provides many features such as calculating priors (mean, standard deviation, and parameters) and provides an open forum for discussion of technical issues.

\section{DOSE ESCALATION SCHEME USING WEB- BASED ONLINE CALCULATOR}

In a prior study, a dose-escalation scheme using EWOC was conducted to design a phase I clinical trial that involves the R115777 drug at Fox Chase Cancer Center in Philadelphia, PA in 1999. We will use this study as an example to demonstrate the application of web-based online dose finding calculator. R115777 is a selective nonpeptidomimetic inhibitor of farnesyltransferase (FTase), one of several enzymes responsible for post-translational modification that is required for the function of p21(ras) and other proteins. It was a repeated-dose, single-center trial designed to determine the MTD of R115777 in patients with advanced incurable cancer. NCIC Expanded Common Toxicity Criteria was used to assess toxicity in the trial. Dose limiting toxicity (DLT) was defined by grade 3 nonhematological toxicity (with the exception of alopecia or nausea/vomiting) or hematological grade 4 toxicity with a possible, probable or likely causal relationship to administration of R115777. Patient participation ended when the occurrence of tumor progression or DLT leading to permanent discontinuation was found. The minimum and maximum dose for this study was $60 \mathrm{mg} / \mathrm{m}^{2}$ and 1000 $\mathrm{mg} / \mathrm{m}^{2}$, respectively. The pilot study showed that five patients receiving each individual dose at 100, 150, 200, 250, or $300 \mathrm{mg} / \mathrm{m}^{2}$ had no toxicity observed. The probability of

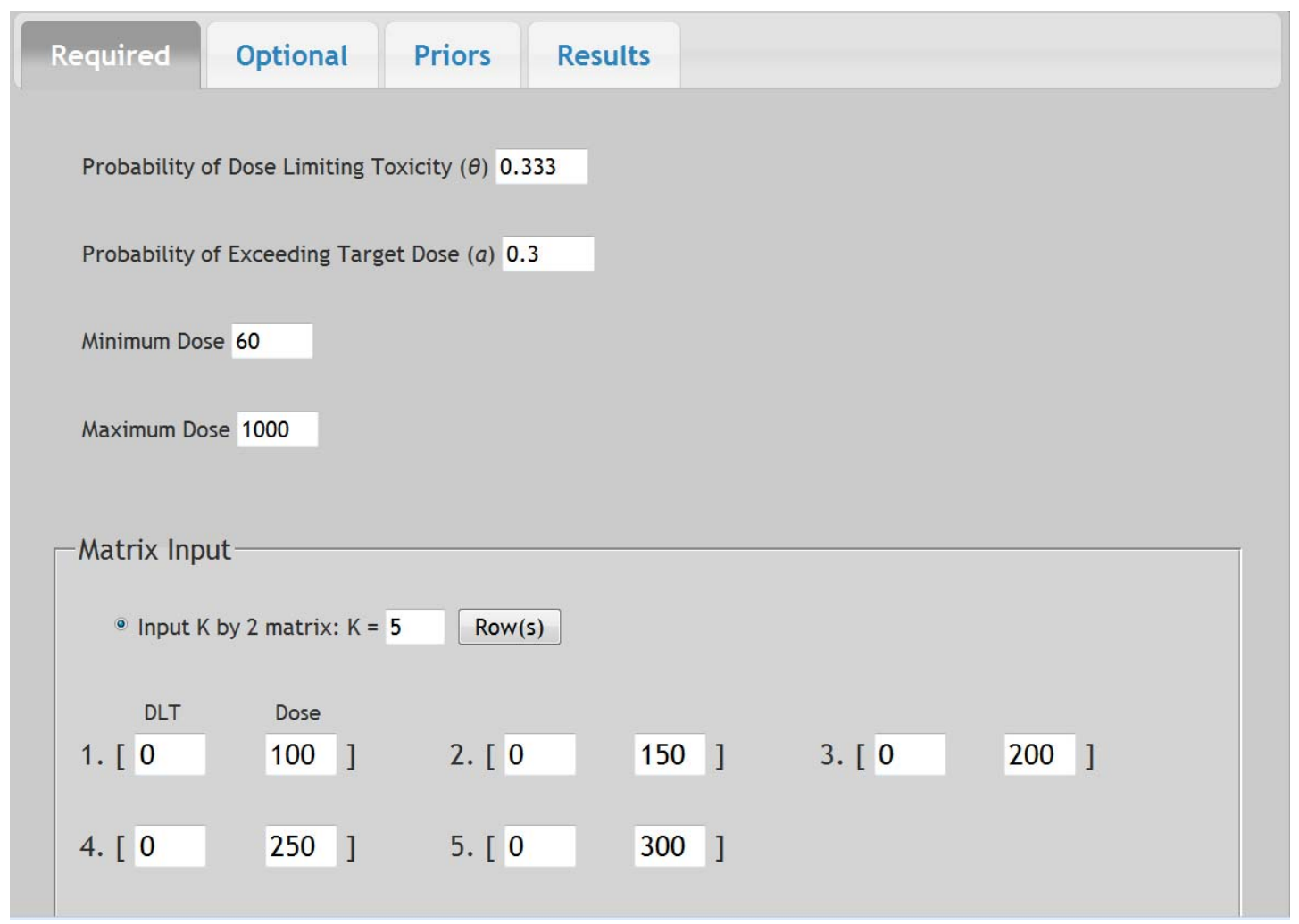

Fig. (4). Screen shot of required parameters. 


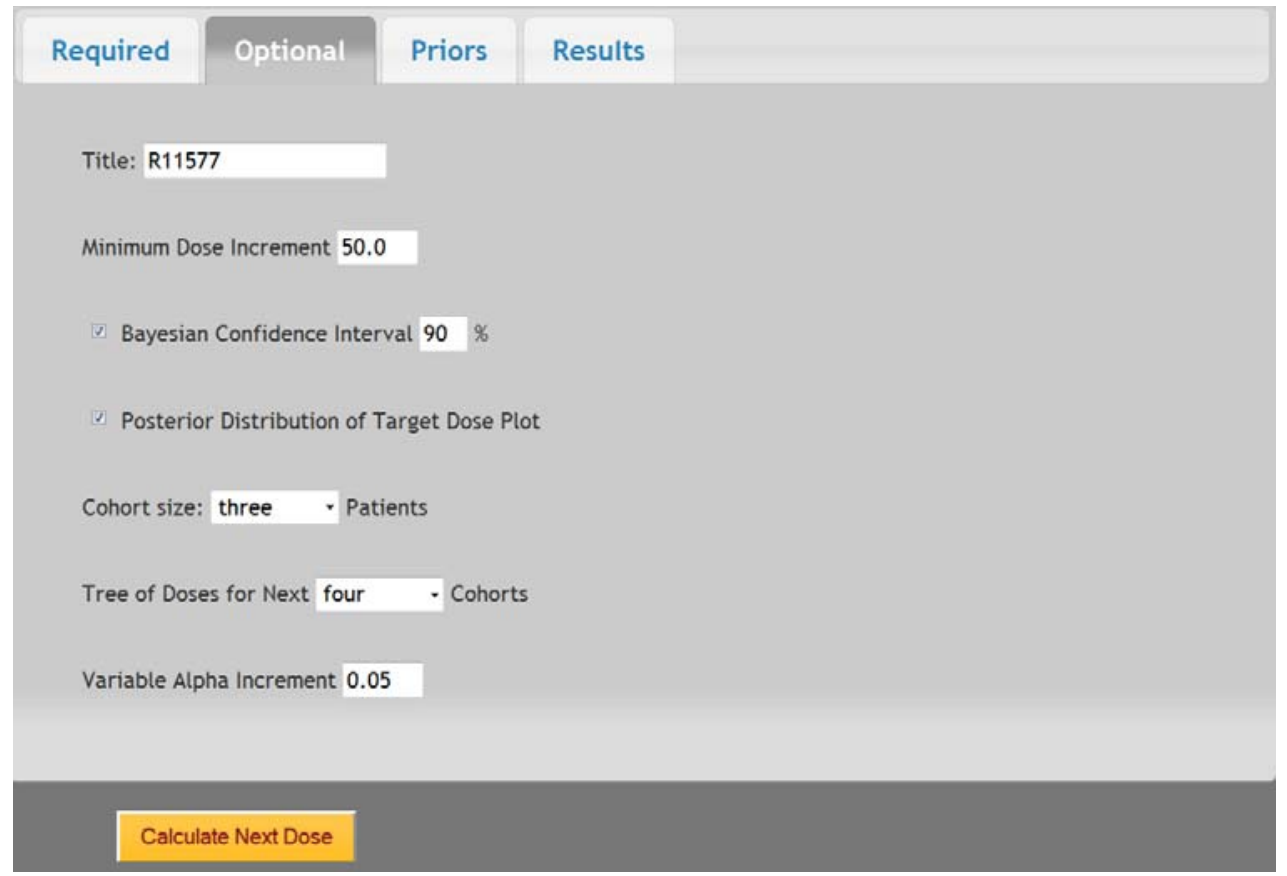

Fig. (5). Screen shot of optional parameters.

DLT at MTD was set as $\theta=0.333$ and the initial probability of a patient exceeding the target dose as $\alpha=0.3$. The drug was supplied in $50 \mathrm{mg}$ and $100 \mathrm{mg}$ capsules, so the minimum dose increment was set as $50 \mathrm{mg} / \mathrm{m}^{2}$. The tree of dose was extended to next five patients. Variable Alpha Increment was set as 0.05 so the feasibility bound $\alpha$ would increase by 0.05 for each patient until it reached 0.50 , the upper limit for the probability of exceeding target dose. The default prior distribution for target dose is uniform distribution between 0 and $\theta$ which is 0.333 here.

Once all parameters are entered in the web-portal based version of EWOC, the user can click the Submit button at the

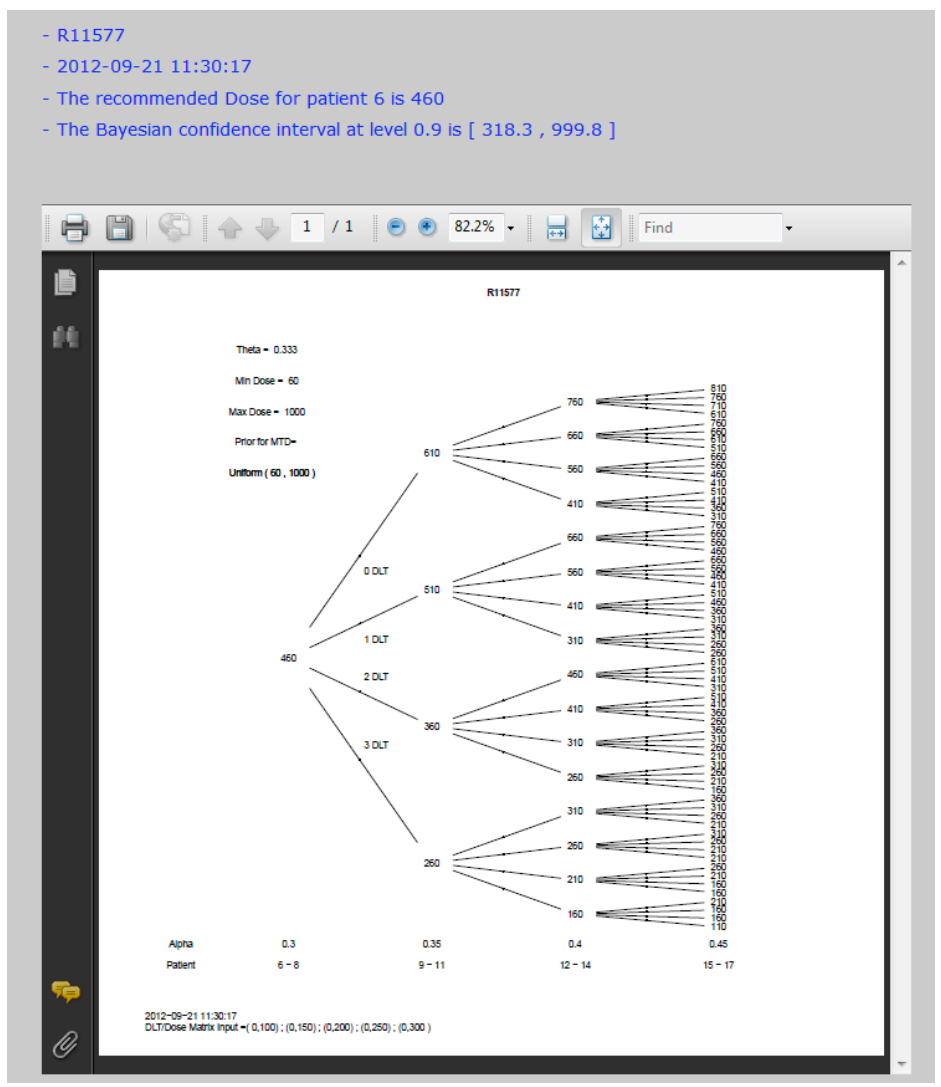

Fig. (6). Dose tree of running example. 
bottom of that page to execute the dose finding calculator. All parameters will be saved in a text file and sent to the Apache web server to invoke the EWOC core program. The computation results will be sent back to web browser for display. Fig. (4) shows the screen shot of required parameters and Fig. (5) shows the screen shot of optional parameters. The dose tree is displayed in Fig. (6).

\section{CONCLUSIONS}

In this paper, we present the design and implementation of a novel web-based online dose finding calculator for cancer phase I clinical trials based on the EWOC algorithm. The online calculator has a user-friendly web interface which can be accessed from any devices such as personal computer or other hand-held devices with standard web browsers. It supports concurrent multi-user access. The running results are stored in the backend database which can be retrieved only by the user. The system is implemented in open source software such as PHP, JQuery, R and OpenBUGS which needs minimal investment and has widely available resources. Currently, only the core algorithm of EWOC has been implemented on the online calculator. In the future, several extensions of the EWOC method such as richer classes of priors, time-to-event and inclusion of covariates will be implemented and integrated into the current web portal.

\section{CONFLICT OF INTEREST}

The authors confirm that this article content has no conflict of interest.

\section{ACKNOWLEDGEMENTS}

Supported in part by the National Center for Research Resources, Grant UL1RR033176, and is now at the National Center for Advancing Translational Sciences, Grant UL1TR000124 (M.T and A.R), grant 5P01CA098912-05 (A.R) and P01 DK046763 (A.R).

The content is solely the responsibility of the authors and does not necessarily represent the official views of the NIH.

\section{REFERENCES}

[1] Rogatko A, Bass JS, Tighiouart M, et al. New paradigm in dosefinding trials: patient-specific dosing and beyond phase I. Clin Cancer Res 2005; 11:5342-6.

[2] Babb J, Rogatko A, Zacks S. Cancer phase I clinical trials: efficient dose escalation with overdose control. Stat Med 1998; 17:1103-20.

[3] Xu Z, Tighiouart M, Rogatko A. EWOC 2.0: interactive software for dose escalation in cancer phase I clinical trials. Drug Inf J 2007; 41(2): 221-8

[4] Zacks, S, Rogatko A, Babb J. Optimal Bayesian-feasibile dose escalation for cancer phase I trials. Stat Probab Lett 1998; 38: 21520 .

[5] Babb J, Rogatko A. Patient specific dosing in a cancer phase I clinical trial. Stat Med 2001; 20(14): 2079-90.

[6] Tighiouart M, Rogatko A, Babb J. Flexible Bayesian methods for cancer phase I clinical trials. Dose escalation with overdose control. Stat Med 2005; 24(14): 2183-96.

[7] Tighiouart M, Rogatko A. Dose Finding with Escalation with Overdose Control (EWOC) in Cancer Clinical Trials. Stat Sci 2010; 25(2): 217-26.

[8] Wooley PV, Schein PS. Methods Cancer Research. New York: Academic Press 1979.

[9] Gatsonis C, Greenhouse JB. Bayesian methods for phase I clinical trials. Stat Med 1992; 11: 1377-89. 